\title{
Majority and minority intrinsic defects in lithium and sodium halides
}

\author{
RADHA D BANHATTI and Y V G S MURTI* \\ Department of Physics, Indian Institute of Technology, Madras 600036 , India \\ * Present address: Department of Physics, Indian Institute of Technology, Guwahati 781 001, \\ India
}

\begin{abstract}
We present new findings on the enthalpies of formation of vacancies and interstitial ion defects on either sublattice in the alkali halide crystals $\mathrm{MX}(X=\mathrm{F}, \mathrm{Cl}, \mathrm{Br}$ and $\mathrm{M}=\mathrm{Li}, \mathrm{Na})$ using the MPPI model. The model uses a minimum fit to crystal properties and is fully consistent with the dielectric response. The role of the non-Coulombic short range forces vis-a-vis polarization fields in the stabilization of the defect formation process is examined and discussed in detail. It is shown that it is important to treat the electrostatic polarization caused by the point defect carefully and variation in potential modelling has a comparatively minor effect on the calculations of enthalpies of formation.
\end{abstract}

Keywords. Vacancies; interstitials; formation enthalpies; alkali halides; crystal potential models; polarization models.

\section{Introduction}

Charge and matter transport studies in the crystals of the alkali halide family have been invariably interpreted in terms of a dominant Schottky disorder without any interstitial component on either sublattice. In the light of the current levels of sophistication in the measurement techniques as well as in the analysis of transport data, it has been recently speculated that there could be a sizeable contribution from interstitials even in these materials (Jacobs and Vernon 1994). The formation enthalpy of the Schottky defect in alkali halides is well quantified both by experiments and theoretical calculations. However the bulk of these calculations overstress the role of the potential parameters, which are found by extensive fitting to crystal properties (Sangster et al 1978).

In this paper we present our findings on the formation enthalpies of the Schottky as well as Frenkel defects, using the modified polarizable point ion (MPPI) model (Murti and Banhatti 1993) and a two-body short-range potential model, whose parameters are minimally fitted to crystal properties. We present detailed comparison on $\mathrm{NaCl}$ with a previous work also based on MPPI model (Murti and Usha 1976; Usha 1976) but using the Tosi potential (Tosi 1964). By this attempt we wish to highlight the larger importance of the polarization model for ionic crystals as against subtle refinements in the potential model.

\section{Potential models}

In the construction of a two-body potential model with the short-range interactions given by the expression

$$
\phi_{i j}(r)=A_{i j} \exp \left[-\frac{r_{i j}}{\rho_{i j}}\right]-\frac{C_{i j}}{r_{i j}^{6}},
$$

\footnotetext{
*Author for correspondence
} 
we need to obtain nine parameters, namely the repulsion parameters $A_{i j}$ and $\rho_{i j}$ and the van der Waals (vdW) coefficients $C_{i j}$. The short range parameters were obtained in the shell model schemes by fitting to six independent bulk properties, namely three elastic constants, lattice constant $r_{0}$ and the static or high frequency dielectric constants as well as the transverse optical phonon frequency. Of these, the elastic constant equations use the first and second derivatives of the potential and to extract each of the potential parameters without introducing arbitrariness is difficult. The vdW parameters are taken from Mayer's (1933) type of calculations.

Alkali halides are simpler to deal with since they are closed shell ionic systems. The Coulomb part is well represented by the Madelung potential. For the non-Coulombic part, one can then use the calculations of the type made by Gordon and Kim (1972) (GK), Cohen and Gordon (1979) and Macrodt and Stewart (1979), which are based on an electron gas model. The correlation energy is however poorly estimated by this model. Hence, we have been constrained to consider the GK data for lithium and sodium halides, where we expect the vdW type of forces to be small and can be easily identified and eliminated when one fits the data to an exponential form. Using the values of $A_{i j}$ and $\rho_{i j}$ in the equilibrium lattice condition, as well as the interrelation between the $C_{i j} \mathrm{~s}$, we determine the vdW parameters. These correspond to the Set III parameters of our potential model (Banhatti et al 1991). Working within the MPPI scheme, we determine the electronic polarizabilities $\left(\alpha_{c}\right.$ and $\left.\alpha_{a}\right)$ using the experimental value of the static dielectric constant and the displacement polarizability $\alpha_{d}$ determined from the short range interactions (Banhatti and Murti 1993). These are tabulated in tables 1 and 2.

The vdW coefficients of the lithium halides turn out to be quite negligible as expected and are hence set to zero. The values of $\alpha_{c, a}$ are small in the lithium halides and $\alpha_{d}$ are large. These may well result in larger ion relaxations around the defects.

Table 1. Overlap repulsion parameters (electron gas model).

\begin{tabular}{lcccrrr}
\hline Crystal & LiF & LiCl & LiBr & NaF & NaCl & \multicolumn{1}{c}{ NaBr } \\
\hline$A_{\text {CC }}\left(10^{3} \mathrm{eV}\right)$ & 2.0260 & 2.0260 & 2.0260 & 45.7200 & 45.7200 & 45.7200 \\
$A_{\text {CA }}\left(10^{3} \mathrm{eV}\right)$ & 0.6724 & 0.7901 & 0.8713 & 2.5504 & 1.7363 & 1.8256 \\
$A_{\text {AA }}\left(10^{3} \mathrm{eV}\right)$ & 2.2431 & 1.2272 & 2.9482 & 2.2431 & 1.2272 & 2.9482 \\
$\rho_{\text {Cd }}(\AA)$ & 0.1215 & 0.1215 & 0.1215 & 0.1422 & 0.1422 & 0.1422 \\
$\rho_{\text {CA }}(\AA)$ & 0.2508 & 0.2991 & 0.3144 & 0.2435 & 0.3049 & 0.3193 \\
$\rho_{\text {AA }}(\AA)$ & 0.2494 & 0.3214 & 0.3320 & 0.2494 & 0.3214 & 0.3320 \\
\hline
\end{tabular}

Table 2. van der Waals coefficients and dipole polarizabilities. C's are in eV $\AA^{6}$ and $\alpha^{\prime}$ s are in $\AA^{6}$.

\begin{tabular}{lcccccc}
\hline Crystal & $C_{\mathrm{C}}$ & $C_{\mathrm{CA}}$ & $C_{\mathrm{AA}}$ & $\alpha_{\mathrm{C}}$ & $\alpha_{\mathrm{A}}$ & $\alpha_{\mathrm{d}}$ \\
\hline $\mathrm{LiF}$ & 0.00 & 0.00 & 0.00 & 0.002 & 0.028 & 1.378 \\
$\mathrm{LiCl}$ & 0.00 & 0.00 & 0.00 & 0.017 & 0.465 & 2.864 \\
$\mathrm{LiBr}$ & 0.00 & 0.00 & 0.00 & 0.032 & 0.032 & 3.341 \\
$\mathrm{NaF}$ & 1.307 & 0.517 & 0.812 & 0.100 & 0.294 & 1.448 \\
$\mathrm{NaCl}$ & 3.725 & 3.693 & 13.758 & 0.149 & 1.032 & 2.607 \\
$\mathrm{NaBr}$ & 5.327 & 6.119 & 28.341 & 0.181 & 1.563 & 3.045 \\
\hline
\end{tabular}


Table 3. Energy contributions (eV) to the defect formation enthalpy in $\mathrm{NaCl}$.

\begin{tabular}{|c|c|c|c|c|c|c|c|c|}
\hline \multirow[b]{4}{*}{ Defect } & \multicolumn{4}{|c|}{ Vacancy } & \multicolumn{4}{|c|}{ Interstitial } \\
\hline & \multicolumn{2}{|c|}{ Cation } & \multicolumn{2}{|c|}{ Anion } & \multicolumn{2}{|c|}{ Cation } & \multicolumn{2}{|c|}{ Anion } \\
\hline & (1) & (2) & (1) & (2) & (1) & (2) & (1) & (2) \\
\hline & $s_{*}$ & $s_{\mathbf{a}}$ & $s_{\mathrm{c}}$ & $s_{\mathrm{c}}$ & $s_{\mathrm{a}}$ & $s_{\mathrm{a}}$ & $s_{\mathrm{c}}$ & $s_{\mathrm{c}}$ \\
\hline$s_{\mathrm{a} / \mathrm{c}}$ & 0.08 & 0.08 & 0.09 & 0.09 & 0.14 & 0.16 & $0-18$ & 0.19 \\
\hline$W_{-1}$ & 8.02 & 7.95 & 8.00 & $8 \cdot 10$ & 2.07 & 2.98 & $4 \cdot 27$ & $4 \cdot 42$ \\
\hline$W_{\mathrm{co}}^{\mathrm{i}}$ & -2.06 & -2.06 & $-2 \cdot 27$ & $-2 \cdot 27$ & -3.00 & -3.32 & $-3 \cdot 51$ & -3.63 \\
\hline$W_{\mathrm{sr}}^{\text {rep }}$ & 1.01 & 1.08 & $1 \cdot 22$ & $1 \cdot 21$ & $1 \cdot 14$ & 0.79 & -0.38 & -0.28 \\
\hline$W_{\mathrm{sr}}^{\mathrm{vdw}}$ & -0.04 & -0.05 & -0.05 & -0.06 & 0.03 & -0.04 & 0.17 & 0.13 \\
\hline$W_{p}^{1}$ & -0.06 & 0.05 & 0.21 & 0.18 & -0.20 & -0.08 & -0.36 & -0.36 \\
\hline$W_{\mathrm{p}}^{2}$ & $-1 \cdot 70$ & -1.73 & -1.79 & -1.77 & -1.57 & -1.57 & -1.57 & -1.57 \\
\hline$W_{\mathrm{d}}$ & $5 \cdot 20$ & $5 \cdot 23$ & $5 \cdot 29$ & $5 \cdot 37$ & -1.68 & -1.42 & -1.71 & -1.30 \\
\hline
\end{tabular}

Table 4. Defect formation enthalpies (eV).

\begin{tabular}{lllllll}
\hline Defect enthalpy & $\mathrm{LiF}$ & $\mathrm{LiCl}$ & $\mathrm{LiBr}$ & $\mathrm{NaF}$ & $\mathrm{NaCl}$ & $\mathrm{NaBr}$ \\
\hline$h_{\mathrm{S}}(\mathrm{eV})$ & $2 \cdot 65$ & $1 \cdot 83$ & $1 \cdot 59$ & $3 \cdot 25$ & $2 \cdot 48$ & $2 \cdot 22$ \\
$h_{\mathrm{S}}(\mathrm{eV})$ & $2 \cdot 3-2 \cdot 7$ & $2 \cdot 12$ & $1 \cdot 80$ & $2 \cdot 9-3 \cdot 2$ & $2 \cdot 4-2 \cdot 5$ & $2 \cdot 2-2 \cdot 4$ \\
$h_{\mathrm{cF}}(\mathrm{eV})$ & $3 \cdot 83$ & $2 \cdot 60$ & $2 \cdot 40$ & $4 \cdot 69$ & 3.52 & $3 \cdot 26$ \\
$h_{\mathrm{aF}}(\mathrm{eV})$ & $4 \cdot 75$ & $3 \cdot 16$ & $3 \cdot 84$ & $5 \cdot 06$ & $3 \cdot 58$ & $4 \cdot 14$ \\
\hline
\end{tabular}

\section{Defect formation energies}

Working within the MPPI scheme of polarization, we have estimated the formation energies for the different defects using two potential sets. The various energy contributions are shown in table 3. The column (1) corresponds to our recent Set III potential and the column (2) of the results corresponds to the potential of Murti and Usha (1976).

We note that the equilibrium displacements $\left(s_{\mathrm{a}}\right.$ or $\left.s_{\mathrm{c}}\right)$ hardly change from one potential set to the other in the case of the vacancy defects. The differences in most of the terms (the rigid lattice energy $W_{\mathrm{r} 1}$, the Coulomb term $W_{\mathrm{co}}^{1}$, the short range terms $W_{\mathrm{sr}}$ and the second region polarization energy $W_{\mathrm{p}}^{2}$ ) are merely marginal and do not finally affect the vacancy formation energy $W_{\mathrm{d}}$ in any significant way. One term where there is a marked change is the region 1 polarization energy $W_{\mathrm{p}}^{\mathbf{1}}$ where as a result of potentials, the dipole polarizabilities are different. In the case of the cation interstitial, the effect of differences in short range potentials is likely to be more pronounced since the defect is contained in a smaller volume of region 1 and hence there exist marked differences in some of the energy terms.

The formation enthalpies of Schottky disorder $\left(h_{\mathrm{S}}\right)$, cationic Frenkel disorder $\left(h_{\mathrm{cF}}\right)$ and anionic Frenkel disorder $\left(h_{\mathrm{aF}}\right)$ are shown in table 4 for the six salts, along with the experimental values of $h_{\mathrm{s}}$. It may be seen that the calculated values of $h_{\mathrm{s}}$ obtained using the MPPI model are in good agreement with the experimental values. This suggests that with the low effective dipole polarizabilities of ions in these salts, the MPPI model is adequate. Further, the minority defect enthalpies are much larger than the majority 
defect enthalpy by values ranging between $0 \cdot 8 \mathrm{eV}-2 \cdot 0 \mathrm{eV}$. This is a significant finding which makes the possibility of the minority defects playing a significant role in transport processes in these crystals quite remote.

\section{References}

Banhatti R D and Murti Y V G S 1993 Phys. Rev. B48 6839

Banhatti R D, Murti Y V G S and Laskar A L 1991 Phys. Status Solidi b164 357

Cohen A J and Gordon R G 1975 Phys. Rev. B12 3228

Gordon R G and Kim Y S 1972 J. Chem. Phys. 563122

Jacobs P W M and Vernon M L 1994 VII Europhysical conf. on defects in insulating materials, Lyon, France Macrodt W C M and Stewart R F 1979 J. Phys. C12 431

Mayer J E 1933 J. Chem. Phys. 1327

Murti Y V G S and Usha V 1976 Physica B83 275

Murti Y V G S and Banhatti R D 1993 Proc. int. conf. in insulating materials (eds) O Kanert and J-M Spaeth (Singapore: World Scientific) p. 198

Sangster M J L, Schroder U and Atwood R M 1978 J. Phys. 111

Tosi M P 1964 in Solid state physics (eds) F Seitz and D Turnbull (New York: Academic Press) 16 p. 1

Usha V 1976 Theoretical studies on the formation and interaction of point defects in ionic crystals, Ph.D. Thesis. IIT Madras 